CYBERNETICS AND INFORMATION TECHNOLOGIES • Volume 16, No 6 Special issue with selection of extended papers from 6th International Conference on Logistic, Informatics and Service Science LISS'2016

Sofia $\bullet 2016$

\title{
Indirect Detection Method of Rotor Position Based on DE-SVM
}

\author{
Bo Wang ${ }^{1}$, Xiaofu $\mathrm{Ji}^{1}$, Jihe Cai ${ }^{2}$ \\ ${ }^{1}$ School of Electrical and Information Engineering, Jiangsu University, Zhenjing 212003, China, \\ ${ }^{2}$ School of Electrical and Photoeletronic Engineering, Changzhou Institute of Technology, Changzhou \\ 213022, China \\ Emails:wangbo@ujs.edu.cn xiaofuji@gmail.com wbwbwb8713@163.com
}

\begin{abstract}
In view of the defects and deficiencies of existing detection methods of rotor position for Switched Reluctance Motor (SRM), an indirect Detection Method (DM) based on DE-SVM for Support Vector Machine (SVM) rotor position is proposed. This method uses the three-phase current and flux linkage within the full angle domain of SRM as input and rotor position angle as output, and utilizes the strong nonlinear mapping capability of SVM to create a predication model for these three parameters offline. The strong global optimization capability of Differential Evolution (DE) Algorithm is then employed based on the deviation between actual rotor position and model output to optimize the prediction model online, thereby realizing sensorless detection of SRM rotor position. The simulation result shows that this method can accurately predict the position of SRM rotor.
\end{abstract}

Keywords: SRM, rotor position detection, SVM, DE Algorithm.

\section{Introduction}

Rotor position detection is the main supporting technology for SRM's (Switched Reluctance Motor) speed governing system and plays a vital role for the safe and reliable operation of the whole system. Researchers have been devoted to the study of this field since the stepping motor research team in the University of Leeds first created the prototype of SRM in 1970. Although rotor position detection technology is maturing increasingly and researchers have proposed a variety of detection schemes, this does not mean that rotor position detection technology is perfect. One of the ultimate goals sought by researchers is to indirectly detect rotor position without using position sensor.

At present, the indirect detection method for SRM rotor position generally achieves this goal by inputting a low voltage detection signal into non-energized winding or by detecting the phase voltage and phase current of SRM without using 
additional signal [1]. However, during actual operation, these methods are subject to a number of problems [2] and have difficulty in providing accurate position of SRM rotor when the motor works in low speed, high speed or transition state. Because of this, some researchers applied artificial intelligence theory to SRM according to its electromagnetic relation and basic characteristics to indirectly detect rotor position. Based on the analysis of SRM's $\Psi-I-\theta$ relation curve, in [3] is proposed a method for indirectly detecting SRM rotor position based on fuzzy logic algorithm, which provides a new idea for indirect detection of rotor position. After that, several improved algorithms were proposed successively [4-6]. However, these algorithms failed to fully consider the inherent defects and deficiencies of fuzzy logic. For example, the algorithm itself has difficulty in ensuring experimental knowledge acquisition, knowledge expression and study, etc., in performing strict theoretical analysis on some of SRM's dynamic characteristics (stability, robustness, etc.) during its operation, and so on. On the basis of the good performance of Artificial Neural Networks in system identification, the flux linkage characteristic data of a 3-phase 6/4-pole SRM model is used in [7] as sample to create a BP neural network with flux linkage and current as input and rotor position angle as output, which was simulated and verified in MATLAB. In [8] a new SRM detection method is proposed without position sensor based on adaptive Radial Basis Function (RBF) neural network, which was simulated and experimentally verified. In [9], two different wavelet neural networks are used to establish nonlinear mapping of current, flux linkage and switching signal, thus realizing indirect detection of motor position. Since the neural networks used by these methods are based on traditional statistical theory (which studies the asymptotic theory when number of samples approach infinity), and SRM is of a doubly salient structure, the motor often works in a deep magnetic saturation state in order to improve motor output power density, thus leading to high nonlinearity of SRM's electromagnetic characteristics. For this reason, its sample data are subject to significant system error due to the influence of instrument and operating procedure. In addition, there are still some problems associated with the intelligent control of neural networks, such as selection of model structure, convergence of algorithm and uniqueness of solution. In recent years, with the continuous development of Support Vector Machine (SVM) theory [10], new ideas for SRM rotor position detection are also emerging. SVM is a kind of machine learning method based on statistical learning theory and structural risk minimization principle [11]. As compared to traditional learning methods such as fuzzy logic and neural network, SVM features a more solid theoretical basis and a better generalization capability even with a smaller number of samples. SVM online training algorithm also features simplicity, short operation time, strong system dynamic response and a certain degree of adaptability, so it is suitable for solving SRM which is a nonlinear system [12].

On the basis of the above, this paper proposes a new sensorless position detection method based on DE-SVM, which uses SVM method within the full angle domain of an SRM to create a nonlinear prediction model for three-phase current, flux linkage and rotor position; Differential Evolution (DE) algorithm is employed to perform online optimization on model parameters according to the model's 
deviation signal, in order to realize indirect detection of SRM rotor position. This method was also simulated and analysed in MATLAB/SIMULINK environment and the simulation result demonstrates its effectiveness.

\section{DE-SVM modelling algorithm}

\subsection{SVM Algorithm}

There are a number of SVM algorithms available. This paper employs the Least Squares Support Vector Machine (LS-SVM) Algorithm. LS-SVM Algorithm changes the inequality constraint condition in a standard SVM Algorithm to equality constraint, and replaces the original relaxation factor with error sum of squares, thus simplifying and making the solving process faster. The LS-SVM optimization problem is given as follows:

$$
\begin{aligned}
& \min J(\boldsymbol{w}, b, \boldsymbol{\xi})=\frac{1}{2} \boldsymbol{w}^{\mathrm{T}} \boldsymbol{w}+\gamma \frac{1}{2} \sum_{i=1}^{l} e_{i}^{2}, \\
& \text { s.t. } y_{i}=\boldsymbol{w}^{\mathrm{T}} \varphi\left(\boldsymbol{x}_{i}\right)+b+e_{i}, i=1,2, \cdots, l,
\end{aligned}
$$

where $\varphi\left(\boldsymbol{x}_{i}\right)$ is kernel space mapping function, $\boldsymbol{w}$ is weight vector, $b$ is threshold parameter, $e_{i}$ is error variance, and $\gamma$ is penalty parameter.

To solve the optimization problem in Equation (1), the following Lagrange function is constructed:

$$
L(\boldsymbol{w}, b, e, \boldsymbol{a})=J(\boldsymbol{w}, b, e)-\sum_{i=1}^{l} a_{i}\left(\boldsymbol{w}^{\mathrm{T}} \varphi\left(\boldsymbol{x}_{i}\right)+b+e_{i}-y_{i}\right),
$$

where $\boldsymbol{a}=\left(a_{1}, a_{2}, a_{3}, \cdots, a_{l}\right)^{\mathrm{T}} \in \boldsymbol{R}^{l}$.

According to KKT conditions:

(3)

$$
\left\{\begin{array}{l}
\frac{\partial L}{\partial \boldsymbol{w}}=0 \Rightarrow \boldsymbol{w}=\sum_{i=1}^{l} a_{i} \varphi\left(\boldsymbol{x}_{i}\right), \\
\frac{\partial L}{\partial b}=0 \Rightarrow \sum_{i=1}^{l} a_{i}=0, \\
\frac{\partial L}{\partial e_{i}}=0 \Rightarrow a_{i}=C e_{i}, \quad i=1,2, \cdots, l, \\
\frac{\partial L}{\partial a_{i}}=0 \Rightarrow w^{\mathrm{T}} \varphi\left(\boldsymbol{x}_{i}\right)+b+e_{i}-y_{i}=0, \quad i=1,2, \cdots, l .
\end{array}\right.
$$

Reorganizing that equation to remove $e_{i}$ and $\boldsymbol{w}$, the optimization problem is transformed into solving the following equation:

$$
\left[\begin{array}{cc}
0 & \boldsymbol{E}^{\mathrm{T}} \\
\boldsymbol{E} & \boldsymbol{K}+\gamma^{-1} \boldsymbol{E}
\end{array}\right]\left[\begin{array}{l}
b \\
\boldsymbol{a}
\end{array}\right]=\left[\begin{array}{l}
0 \\
\boldsymbol{y}
\end{array}\right],
$$


where $y=\left[y_{1}, y_{2}, y_{3}, \cdots, y_{l}\right]^{\mathrm{T}}, \boldsymbol{E}=[1,1,1, \cdots, 1]^{\mathrm{T}}$, and $\boldsymbol{K}=K\left(\boldsymbol{x}_{i}, \boldsymbol{x}_{j}\right)=\varphi^{\mathrm{T}}\left(\boldsymbol{x}_{i}\right) \varphi\left(\boldsymbol{x}_{j}\right)$ are kernel functions; RBF kernel function $K\left(\boldsymbol{x}_{i}, \boldsymbol{x}_{j}\right)=\exp \left[-\left|\boldsymbol{x}_{i}-\boldsymbol{x}_{j}\right|^{2} /\left(2 \sigma^{2}\right)\right]$ is selected in this paper. The following LS-SVM expression is obtained:

$$
f(\boldsymbol{x})=\sum_{i=1}^{l} a_{i} K\left(\boldsymbol{x}_{i}, \boldsymbol{x}_{j}\right)+b .
$$

For LS-SVM algorithm, the selection of kernel function parameter $\sigma$ and penalty parameter $\gamma$ has an important influence on the creation of a nonlinear model. Traditional parameters are generally selected based on experience using the trial-and-error method, with which accuracy and computing speed are difficult to be ensured.

To obtain a nonlinear model capable of giving better prediction result, this paper uses DE Algorithm to perform online optimization and adjustment on LSSVM parameters. In recent years, many scholars proposed some other parameter optimization methods. For example, in [13] Gradient Descend (GD) method is used for parameter optimization, which, though greatly shortening the time required for parameter search, has higher requirements on the selection of initial point and is a linear search method easy to fall into local optimum; Particle Swarm Optimization (PSO) algorithm is used in [14]; in [15] ant colony algorithm is used to optimize SVM parameters. Although these optimization methods reduce the dependence on initial parameter selection, they feature complex algorithm principles and ideas, and require designing different crossover, mutation and selection operations for different optimization problems, which are easy to fall into local optimum. DE algorithm was proposed jointly by Rainer Storn and Kenneth Price in 1995 to solve the fitting problem of Chebyshev polynomials, which is an optimization algorithm that uses floating point vector coding to perform random search in a continuous space [16]. DE features simple principles and less controlled parameters. It is easy to understand and implement. As a very effective heuristic evolutionary algorithm, it can implement random, parallel or direct global search and is increasingly noticed and recognized by scholars. Therefore, to obtain a prediction model with higher precision, this paper proposes a combined modelling method based on DE-SVM, which first uses SRM's three-phase winding phase current and phase flux linkage as input, and actual rotor position as output, then employs SVM to create a nonlinear prediction model for SRM's current, flux linkage and rotor position, and finally uses DE Algorithm to optimize and correct the key parameters of SVM, thus improving its prediction accuracy.

\subsection{DE Algorithm}

DE uses a computational procedure similar to a standard evolutionary algorithm and includes three types of operation: mutation, crossover and selection [17]. However, unlike traditional evolutionary algorithms, DE uses the proportional difference vector created from different randomly selected individuals to disturb those in the population of current generation, thus eliminating the need to use a separate probability distribution to create a filial generation. In $\mathrm{DE}$ 
Algorithm, a $D$-dimensional vector containing NP real parameters $X_{i}^{t}=\left\{x_{i 1}^{g}, \cdots, x_{i D}^{g}\right\} \in S(i=1, \cdots, N P) \quad$ forms one generation of population $P^{g}=\left\{X_{i}^{g}, \cdots, X_{N P}^{g}\right\}$ to perform parallel direct search in the search space, where $t=0,1, \cdots, T_{\max }$ is new generation of evolution.

In a mutation operation, a mutated vector $V_{i}^{t}=\left(v_{i 1}^{g}, \cdots, v_{i D}^{g}\right)$ is created after a mutation operation is performed on each target individual, as

$$
V_{i}^{g}=X_{r 1}^{g}+F \cdot\left(X_{r 2}^{g}-X_{r 3}^{g}\right),
$$

where $r_{1}, r_{2}, r_{3}$ are mutually different integers randomly selected from set $\{i=1,2, \cdots, \mathrm{NP}\}$, and $F$ is the scaling factor within the range of $[0.4,1]$, which is used to control the scaling of difference vector.

To improve the potential diversity of the population, a crossover operation is performed on target vector $X_{i}^{g}$ and its mutated vector $V_{i}^{g}$, to create a test vector $U_{i}^{t}=\left(u_{i 1}^{g}, \cdots, u_{i D}^{g}\right)$ :

$$
u_{i j}^{t}=\left\{\begin{array}{cc}
v_{i j}^{t} & \text { if } \operatorname{rand}_{i j} \leq \mathrm{CR} \text { or } j=j_{\text {rand }}, \\
x_{i j}^{t} & \text { otherwise, }
\end{array}\right.
$$

where $\operatorname{rand}_{i j}$ is a uniform random number within the range of $[0,1]$, which determines whether element $j$ of test vector $i$ is offered by the mutated vector or the target vector, $j_{\text {rand }} \in[1,2, \cdots, D]$ is a randomly selected dimension index, which ensures that at least one element in test vector $U_{i}^{t}$ is offered by mutated vector $V_{i}^{g}$, and $\mathrm{CR} \in[0,1]$ is crossover probability constant.

For selection operation, the greedy strategy is adopted, which utilizes the next equation to compare the target function value of the test vector with the target function of the corresponding target individual. If the former is less than or equal to the latter, the test vector replaces the corresponding target individual in the next generation, or the target vector remains unchanged:

$$
X_{i}^{t+1}=\left\{\begin{array}{l}
U_{i}^{g}, \text { if } f\left(U_{i}^{g}\right) \leq f\left(X_{i}^{g}\right), \\
X_{i}^{g} \text { otherwise, }
\end{array}\right.
$$

where $f$ is the target function, which is selected in this paper as the squared error $\min \sum\left(y_{t}^{*}-y_{t}\right)^{2}$ between SVM output value and offline test value, where $y_{t}$ is the true value of position detection in time $t$, and $y_{t}^{*}$ is SVM's predicted value, where $t=1,2, \cdots, l$.

The specific steps for DE-based SVM parameter optimization are as follows:

Step 1. Initialize population size $N$, evolutional generation $g_{\mathrm{m}}$, scaling factor $F$, crossover probability CR, stopping threshold as well as upper and lower limits of SVM penalty parameter $\gamma$ and kernel parameter $\sigma$ to randomly create $(\gamma, \sigma)$.

Step 2. Use the combined value of $(\gamma, \sigma)$ as the parameter of SVM and employs SVM to conduct offline training and test to obtain a test result, i.e., the predicted result from samples. 
Step 3. Compare the predicted result obtained in Step 2 with the actual value, calculate the target function value, and determine whether this value meets the prediction accuracy or satisfies $g=g_{\mathrm{m}}$, i.e., reaches the maximum evolutional generation; if either one is met, go to Step 9, or go to the next step.

Step 4. $g=g+1$, go to the next evolutional generation.

Step 5. Randomly select three different individuals $X_{i}^{g}$ from the population of generation $g$, and perform mutation operation as per Equation (6) to create mutated individuals $V_{i}^{g+1}$ of generation $g+1$.

Step 6. Perform mutation operation on mutated individuals $V_{i}^{g+1}$ of generation $g+1$ as per Equation (7) to create test individuals $u_{i j}^{g+1}$ of generation $g+1$.

Step 7. Perform selection operation on test individuals $u_{i j}^{g+1}$ of generation $g+1$ as per Equation (8) to create individuals of generation $g+1$.

Step 8. In the population of generation $g+1$, calculate new $(\gamma, \sigma)$, and then go to Step 2).

Step 9. Obtain the optimal parameters $(\gamma, \sigma)$ of SVM.

Featuring simple principles, less controlled parameters and fast convergence rate, DE Algorithm is an effective search algorithm for global optimal solution and is suitable for solving complex optimization problems. Taking advantages of DE's strong global optimization capability, this paper performs online optimization and adjustment on SVM's performance parameters $\sigma$ and $\gamma$ to ensure that a more accurate nonlinear model of the system can be obtained.

\subsection{Steps of DE-SVM-Based Modelling Algorithm}

Steps of DE-SVM-Based Modelling Algorithm are as follows:

Step 1. Create sample training data pairs $\left\{\boldsymbol{x}_{t}, y_{t}\right\}, t=1, \cdots, l$, using the threephase current and flux linkage within the full angle domain of an SRM as input and rotor position angle as output, and then normalize the sample data.

Step 2. Employ the crossover verification method to perform SVM offline training to obtain the initial $(\gamma, \sigma)$.

Step 3. Initialize population size $N$, evolutional generation gm, scaling factor $F$, crossover probability CR, stopping threshold as well as upper and lower limits of SVM's penalty parameter $\gamma$ and kernel parameter $\sigma$.

Step 4. Employ DE Algorithm to perform online optimization on SVM to obtain its optimal parameters $(\gamma, \sigma)$ according to the actual rotor position and the deviation signal min $\sum\left(y_{t}^{*}-y_{t}\right)^{2}$ outputted by the SVM model.

Step 5. Obtain penalty parameter and kernel function parameter through optimization, obtain Lagrange factor $a_{i}$ using LS-SVM Algorithm, and then solve Equation (4) to obtain corresponding $a_{i}$ and $b$ to determine SVM's nonlinear prediction model $f(\boldsymbol{x})=\sum_{i=1}^{l} a_{i} K\left(\boldsymbol{x}_{i}, \boldsymbol{x}_{j}\right)+b$. 
Step 6. Evaluate the predicted result: Evaluate the prediction capability of the model by setting a standard deviation (RMSE) as the performance indicator. The calculation formula is

$$
\text { RMSE }=\sqrt{\frac{1}{l-1} \sum_{i=1}^{l}\left|y_{t}^{*}-y_{t}\right|^{2}},
$$

where $y_{t}$ is the true value of SRM rotor position detection in time $t, y_{t}^{*}$ is SVM's predicted value, and $l$ is the number of predicted data.

\section{Simulation experiments and analysis}

While an SRM is running, since the current and magnetic flux in a single-phase turn-off angle remain at zero for a long time, it is difficult to employ DE-SVM algorithm to detect rotor position in a single-phase turn-off angle; the current and magnetic flux in a three-phase motor will not be zero at the same time, so this paper expands the sample data to the full angle domain of a three-phase motor to realize rotor position detection within the motor's full angle domain. Training samples are obtained from SRD model's circuit parameters: supply voltage $U=0.22 \mathrm{kV}$, turnon angle $=2^{\circ}$, turn-off angle $=32^{\circ}$, power $P=1.1 \mathrm{~kW}$, speed $n=1000 \mathrm{rad} / \mathrm{min}$, no load.

The parameters selected in SRM general mode are shown in Table 1:

Table 1. SRM model parameters
\begin{tabular}{|c|c|c|c|c|c|}
\hline $\begin{array}{c}\text { Stator } \\
\text { resistance, } \\
\Omega\end{array}$ & $\begin{array}{c}\text { Moment } \\
\text { of inertia } J, \\
\mathrm{~kg} \cdot \mathrm{m}^{2}\end{array}$ & $\begin{array}{c}\text { Max } \\
\text { inductance, } \\
\mathrm{H}\end{array}$ & $\begin{array}{c}\text { Minductance, } \\
\mathrm{H}\end{array}$ & $\begin{array}{c}\text { Max } \\
\text { current, } \\
\mathrm{A}\end{array}$ & $\begin{array}{c}\text { Max flux } \\
\text { linkage, } \\
\mathrm{Wb}\end{array}$ \\
\hline 0.01 & 0.0082 & 0.0236 & 0.0015 & 450 & 0.486 \\
\hline
\end{tabular}

Data about the 3-phase current, magnetic flux and position angle of an SRM are sampled at motor startup, low speed and high speed, at time intervals and a sampling frequency of $10 \mathrm{kHz}$ to obtain training sample data pairs.

To verify the performance of DE-SVM Algorithm, the predicted result is compared with the actual rotor position. SVM's initial parameters are obtained using crossover verification method: $\sigma^{2}=1.0, \gamma=10$, and optimized SVM's performance parameters are $\sigma^{2}=0.46, \gamma=9.8$.

Fig. 1 shows the comparison diagram for actual rotor position and DE-SVM predicted result at motor startup. Fig. 2 shows the comparison diagram for actual rotor position and DE-SVM predicted result at $50 \mathrm{rad} / \mathrm{s}$. Fig. 3 shows the comparison diagram for actual rotor position and DE-SVM predicted result at $190 \mathrm{rad} / \mathrm{s}$.

As can be seen from Fig. 1, Fig. 2 and Fig. 3, the prediction error is small and the prediction of SRM rotor position is realized at a certain precision. The nonlinear prediction model created using DE-SVM method can give a satisfactory result for rotor position prediction. 


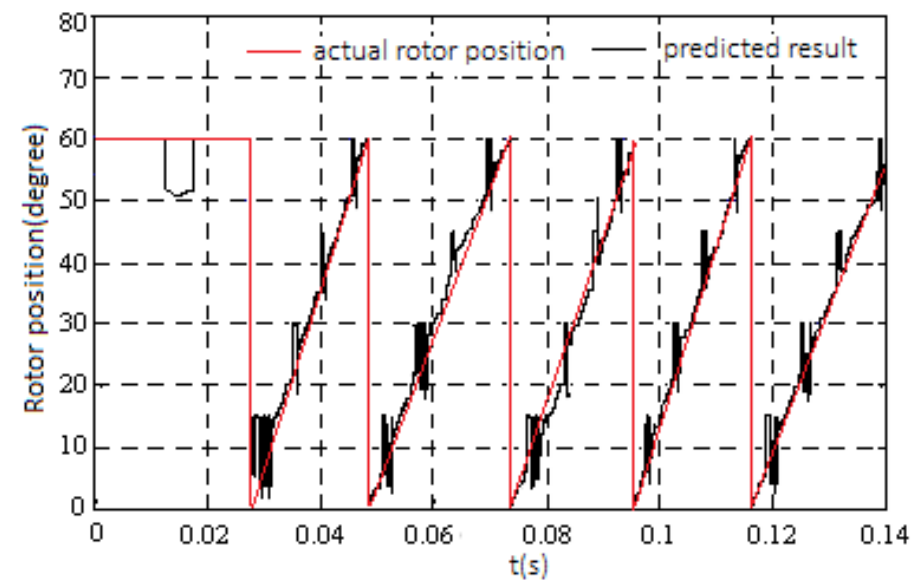

Fig. 1. Comparison diagram for actual rotor position and DE-SVM predicted result at motor startup

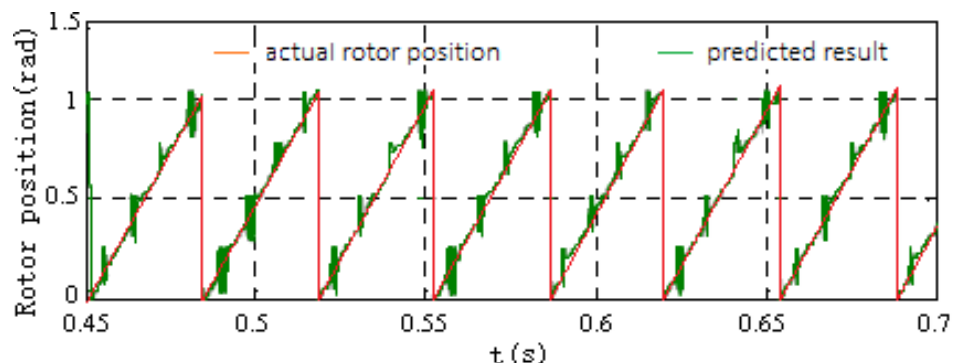

Fig. 2. Comparison diagram for actual rotor position and DE-SVM predicted result at $50 \mathrm{rad} / \mathrm{s}$

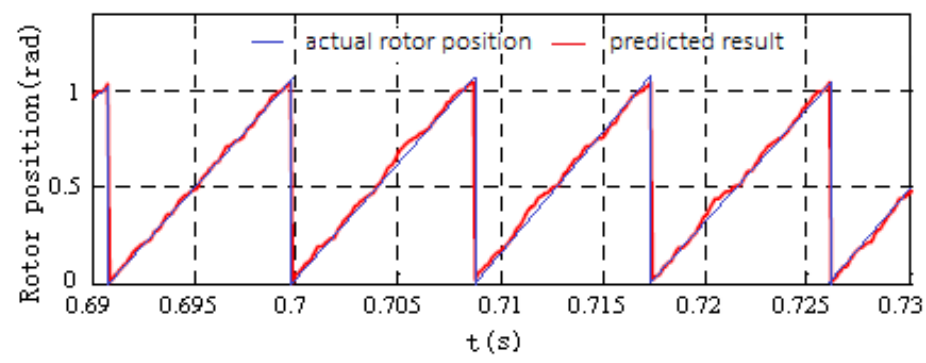

Fig. 3. Comparison diagram for actual rotor position and DE-SVM predicted result at $190 \mathrm{rad} / \mathrm{s}$

\section{Conclusion}

In this paper, SVM's strong nonlinear mapping capability is used to create a nonlinear predication model for SRM's three-phase current, phase flux linkage and rotor position; then the strong global optimization capability of DE Algorithm is employed based on the deviation between actual rotor position and model output to optimize the prediction model online, thereby realizing sensorless detection of SRM rotor position. The simulation result shows that this method can accurately predict the position of SRM rotor. 


\section{References}

1. W e n, Z. M., B. Li u. Comparison of Some Indirect Rotor-Position Detection Techniques for Switched Reluctance Motors. - SCI/TECH Information Development \& Economy, Vol. 15, 2005, No 18, pp. 199-201.

2. Zhou, S. Y., H. Li n. Rotor Position Detection Techniques of Switched Reluctance Motor without Position Sensor. - Electric Drive, Vol. 36, 2006, No 2, pp. 8-10.

3. Ho u, X. C., Y. L. Cu i, J. M. R. Li u. Rotor Position Angle Estimation and Speed Control in SRD Using Fuzzy Logic Based Motor Model. - Electric Drive, Vol. 30, 2003, No 5, pp. 15-18.

4. W a n g, X., Y. G a o, C. J. S u. Rotor Position Angle Estimation Research in SRD Using Fuzzy Logic Rule. - Journal of Yanshan University, Vol. 23, 2006, No 6, pp. 518-521.

5. Li a ng, D. L., W. D ing, Z. M. Y u. Modeling for Switched Reluctance Motor Based on Adaptive Network-Based Fuzzy Inference System. - Proceedings of the CSEE, Vol. 9, 2008, No 9, pp. 86-92.

6. Z h a n g, D. P., W. W a n g, L. X. S u. An Improved Sensorless Driving Method of Switched Reluctance Motors Based on Fuzzy Logic. - Modern Electronics Technique, Vol. 18, 2008, No 6, pp. 171-174.

7. Xi a, C. L., M. C. W a n g, T. N. Shi, P. J. Gu o. Position Sensorless Control for Switched Reluctance Motors Using Neural Network. - Proceedings of the CSEE, Vol. 25, 2010, No 13, pp. 123-128.

8. Ku a i, S. Y., X. L. Zh ang, Q. H. W a n g, N. Zh an g. Position Sensorless Control of SRM Using Neural Network. - Electric Machines and Control, Vol. 15, 2011, No 8, pp. 18-22.

9. X i a, C. L., X. M. X i e, T. N. S h i, Y. T i a n. Position Sensorless Control of Switched Reluctance Motor Using Wavelet Neural Networks. -Transactions of China Electrotechnical Society, Vol. 23, 2008, No 7, pp. 33-38.

10. De n g, N., Y. Ti a n. The New Method of Data Mining Based on Support Vector Machine. Science Press, Beijing, 2004.

11. W a n g, X. D., C. J. Z h an g, H. R. Z h a n g. Sensor Dynamic Modelling Using Least Square Support Vector Machines. - Chinese Journal of Scientific Instrument, Vol. 27, 2006, No 7, pp. 730-733.

12. Fang, R. M. Gas Leakage Detection Based on Clustering Support Vector Machine. - Chinese Journal of Scientific Instrument, Vol. 28, 2007, No 11, pp. 2028-2033.

13. Chappelle, O., V. Vapnik, O. Bousquet et al. Choosing Multiple Parameters for Support Vector Machines. - Machine Learning, Vol. 46, 2002, No 1, pp. 131-160.

14. S h a o, X. G., H. Z. Y a n g, G. C h e n. Parameters Selection and Application of Support Vector Machines Based on Particle Swarm Optimization Algorithm. - Control Theory and Applications, Vol. 23, 2006, No 5, pp. 740-743.

15. Z h u a n g, Y., Z. L. B a i, Y. F. X u. Research on Parameters of Support Vector Machine Based on Antcolony Algorithm. - Computer Simulation, Vol. 28, 2011, No 5, pp. 216-219.

16. W u, L. H., Y. N. W ang et al. Environmental/Economic Power Dispatch Problem Using MultiObjective Differential Evolution Algorithm. - Electric Power Systems Research, Vol. 80, 2010, No 9, pp. 1171-1181.

17. W a n g, L., L. P. Li. Fixed-Structure Hœ Controller Synthesis Based on Differential Evolution with Level Comparison. - IEEE Transactions on Evolutionary Computation, Vol. 15, 2011, No 1, pp. 120-129. 